\title{
Translation of Titles of Chinese Costume Dramas from the Perspective of Cultural Differences*
}

\author{
Zhengwu Huang, Hui Jia \\ School of Foreign Languages, East China University of Science and Technology, Shanghai, China \\ Email:1841025460@qq.com
}

How to cite this paper: Huang, Z.W. and Jia, H. (2020) Translation of Titles of Chinese Costume Dramas from the Perspective of Cultural Differences. Open Access Library Journal, 7: e6881.

https://doi.org/10.4236/oalib.1106881

Received: October 5, 2020

Accepted: October 25, 2020

Published: October 28, 2020

Copyright $\odot 2020$ by author(s) and Open Access Library Inc.

This work is licensed under the Creative Commons Attribution International License (CC BY 4.0).

http://creativecommons.org/licenses/by/4.0/

\section{(c) (i) Open Access}

\begin{abstract}
Chinese costume dramas are rich in Chinese cultural elements, but there is an inescapable challenge in the English translation of the titles: cultural differences. Titles of Chinese costume dramas have two main characteristics: implicitness and simplicity, while those of British and American costume dramas are clear and concise. Therefore, this paper proposes that the primary principle for the English translation of titles of Chinese costume dramas is to simplify and crystallize. Titles of Chinese costume dramas are divided into two categories, and specific translation strategies are proposed in this paper. The strategies of free translation, creative translation and borrowing are preferred for implicit titles; transliteration and literal translation, and subtractive translation are adopted for explicit ones.
\end{abstract}

\section{Subject Areas}

Culture, Linguistics

\section{Keywords}

Titles of Costume Drama, Chinese Culture, Cultural Differences

\section{1. 引言}

在中国文化走出去的战略背景下, 国家和各界都在致力于推动中华文化 的国际化进程。讲好中国故事, 更好地向外国友人展示中华文明的博大精深 和独特魅力, 用一种更加合适的方式让国际友人了解并认可中国优秀传统文 化, 是当前文化走出去战略部署中最重要的一环。影视剧作为一种喜闻乐见

${ }^{\star}$ The paper is supported by the Fund for University Students' Innovation and Entrepreneurship (S19124) of East China University of Science and Technology. 
的媒体, 是传播文化和价值观、塑造国家和民族形象的绝佳窗口。在众多的 影视剧题材中, 古装剧蕴含丰富的中国文化元素, 最能体现地道的中国味, 在促进文化交流和传播中具有举足轻重的作用。

片名作为影视剧的 “眼” , 很大程度上影响了作品的经济效益和社会效 益。总体而言, 影视剧剧名主要有四大功能。“第一, 信息传播功能。也就 是突显影片内容, 帮助观众更好地理解原片。第二, 表情功能。奠定影片感 情基调, 以强烈的抒情、惊险或喜剧氛围感染观众。第三, 审美功能。以语 言的优美流畅、生动形象打动观众, 造成观众审美愉悦。第四, 广告功能。 就是吸引观众, 增加票房收人。”[1]因此, 研究古装剧剧名翻译具有战略意 义与实际应用价值。

在中国知网中, 以 “电影片名翻译” 为主题关键词进行检索, 共计得到 相关文献 1184 篇, 而以 “古装剧翻译” 为关键词进行检索, 仅得到 7 篇相关 文献, 其中仅有 2 篇研究中国古装剧名英译。由此可见, 相比于研究成果丰 硕的电影片名翻译研究, 古装剧剧名翻译的研究十分局限。

从研究方向来看, 影视剧名翻译研究主要是基于传播学、社会学、美学 等多元学科交叉或是功能对等理论以及目的论等理论。古装剧名作为一种特 殊文本, 不同于一般性质的专有名词, 其包含丰富文化信息, 故此, 本文将 从一个新的视角一一文化差异视角, 探讨古装剧名翻译如何突破文化差异的 难题, 助力具有中国特色的古装剧在海外实现更好传播。

\section{2. 文化差异在中英古装剧名中的体现}

\section{1. 文化差异}

古装剧中蕴含着丰富的中国文化因素, 但古装剧名翻译中存在着不可逃 避的问题, 即文化差异。英汉两个民族具有不同的思维方式, 连淑能指出, 中国的传统思维方式具有政治伦理型的特征, 西方形成的是一种科学认知型 的思维方式。中国人注重 “天人合一”, 其思维方式具有整体性和意向性, 西方人强调 “主客二分”, 其思维方式具有分析性和对象性。中国人注重 “立 意于像”, 强调直觉, 更喜模糊, 西方人更注重逻辑推理, 强调实证, 更喜 精确[2]。正是这种思维方式上的不同, 加之英汉两个民族在地域和环境上有 很大差异, 中西方形成了各自不同的文化。

多年来, 诸多影视剧走向国外都出现了水土不服的现象。除了影视质量 本身的问题, 一个很关键的原因就是文化差异。“中西文化的差异和冲突是 中国电视剧海外市场拓展的最大障碍” [3]。所以, 古装剧名翻译应该要首先 考虑到剧名中出现的文化元素, 努力突破文化差异的难题。

\section{2. 中国古装剧剧名的特点}

\subsection{1. 委婉含蓄, 用词华美}

1) 善于借用古典诗词。如《知否知否应是绿肥红瘦》出自宋代女词人李 清照的早期词作《如梦令 - 昨夜雨疏风骤》。《锦绣未央》中 “未央”一词 取自《诗经》中《小雅 - 庭燎》 “夜如何其? 夜未央”。 《不负如来不负卿》 
出自仓央嘉措的诗句 “世间安得双全法, 不负如来不负卿。” 《寂寞空庭春 欲晚》出自唐代诗人刘方平的名作《春怨》 “寂寞空庭春欲晚, 梨花满地不 开门”。《花谢花飞花满天》则是语出曹雪芹的《葬花吟》 “花谢花飞花满 天, 红消香断有谁怜? ” 《风囚凰》音同西汉大辞赋家司马相如所写的《风 求凰》。《思美人》出自屈原《九章 - 思美人》 “思美人兮, 揽涕仁眙”。 古典诗词是中国的文化遗产, 是中国古典文化的内核, 也是中华文化博大精 深的标志, 非常切合古装剧这一类影视题材。同时, 古诗词本身就代表一种 意境, 加之其音律朗朗上口, 特别容易吸引观众眼球。

2）选用具有玄幻色彩的字词，如 “仙” “侠” “神” “幻” “邹” “狐” “生” “世”。如《仙剑奇侠传》《怪侠一枝梅》 《太古神王》 《女娲传说 之灵珠》 《轩辕剑》 《幻城》 《琉璃美人繁》 《青丘狐传说》 《三生三世十 里桃花》 《三生三世枕上书》 《三生三世宸汐缘》等。仙侠剧、玄幺剧是中 国古装剧的重要组成部分, 一直以来深受中国观众喜爱。中国观众对 “仙侠 神魔鬼怪” 情有独钟, 鲜明地体现了东方审美的特点, 即 “天人合一”与 “模 糊” 。同时, 这种情愫深深扎根于中国古代传统文化之中。中华文化历史悠 久, “很多流传至今的经典文学作品都记载了大量奇幻绚丽的神话故事以及 半人半妖的形象。” [4]仙侠妖魔等形象承载着中国古代人无穷的想象力和对 大自然的崇拜, 体现着虚幻、模糊、天人合一的中国古代美学思想。

\subsection{2. 表意明了, 直奔主题}

1) 常以人名作剧名。中国古装剧通常都是大男主大女主剧, 因此常选择 以主角名作为剧名, 而且常会在后面加上 “传” “传奇” 等词, 使整个剧名 显得气象宏大, 给主人公的一生增添了传奇色彩, 使观众在心理上就产生对 故事情节跌宕起伏的期待。如《楚乔传》 《甄嬛传》 《皓镧传》 《如懿传》

《芸汐传》 《㹃月传》 《班淑传奇》《陆贞传奇》 《重耳传奇》 《武媚娘传 奇》。

2) 常以朝代, 官职等作为剧名。古装剧除了借助玄幺武侠元素, 王朝起 伏、宫闱秘史也是重要组成部分。如《兰陵王》 《雍正王朝》 《隋唐英雄传》 《大宋提刑官》 《东宫》 《回到明朝当王爷之杨凌传》 《拜见宫主大人》 《双 世宠妃》《公主驾到》《大汉贤后卫子夫》等。“将军” “皇帝” “妃子” 等早已成为历史, 但在中国几千年历史中, 这些人一直都是主角。看到这些 历史词, 观众会觉得陌生又熟悉, 好奇与憧憬。

\section{3. 英美古装剧剧名特点}

1) 通畅简明, 直奔主题。如 PLEBS(《罗马三贱客》)讲述的是古罗马时 代的三名罗马小市民, 他们懒懒散散, 毫无大志, 注定不会留下任何历史痕 迹。以 Plebes 作为剧名, 直接表明了电视剧的主人公是平平常常的小人物, 剧中不会出现史诗故事。

2) 多用人名, 清晰明了。大部分英美古装剧都是改编自历史以及民间传 奇, 旨在重现史诗历史, 所以很多古装剧都是直接使用主人公名作为剧名。 如 Robin Hood(《侠盗罗宾汉》) 讲述的是英国民间传说英雄罗宾汉的故事, 
Merlin(《梅林传奇》)讲述了年轻的梅林大法师如何认识自己的使命、成为亚 瑟王子的亲密朋友共同拯救世界，和他们之间史诗般的英雄故事。 Spartacus(《斯巴达克斯》)讲述的是一名服役于罗马军队的色雷斯战士斯巴达 克斯的传奇故事。这些剧名直接使用主人公人名, 直入主题, 同时这些历史 上充满传奇色彩的名字十分容易激起观众的文化共鸣, 引发观影兴趣。

\section{3. 文化差异视角下古装剧剧名英译}

影视剧片名作为一种特殊文本, 虽然具有专有名词的特点, 但其功能主 要在于吸引观众的眼球, 激发观众的兴趣。影视剧外译最主要的目的是实现 经济价值, 故而译者应定位好受众, 尽量采用满足受众心理诉求和审美期待 的方式进行翻译。

“不同文化背景下的电视受众群体在审美爱好、文化心理等多方面各有 差异” [5]。通过上述讨论, 我们可以大致发现, 关于古装剧名, 西方观众更 容易接受通畅简明, 直奔主题的剧名。因此, 本文认为, 中国古装剧名英译 的主导原则为, 由繁入简, 由模糊虚幻到具体实在。

一般而言，剧名作为电视剧的 “眼”，一方面直接概括电视剧剧情，另 一方面也体现着电视剧的主题。但是，中国古装剧名过于 “用词华丽、讲究 声律对仗和平衡美” [6], 没有清楚地概括剧情, 或者是影视主题。虽然满足 了中国观众的模糊审美，却也让人觉得云里雾里。鉴于此，本文将中国古装 剧名分成两类, 一类为剧名委婉含蓄, 另一类为剧名表意明了, 分别提出英 译策略。

\section{1. 委婉含蓄类}

\subsection{1. 意译}

意译是指 “根据原文的大意，而不作逐字逐句的翻译” [7]。中国古装剧 名中存在大量具有中国文化特色的意象和表达方式，其内涵与美感无法通过 音译或者直译完整传达。通过意译法, 可以以最符合外国观众语言习惯，最 为形象传神的方式传达剧名的含义，展现剧情以及主题。

下面对表 1 中具有代表性的剧名及其英译名进行分析。

\section{表 1. 采用意译策略的原剧名及其英译剧名}

\begin{tabular}{cc}
\hline 原剧名 & 英译剧名 \\
\hline 幻城 & Ice Fantasy \\
暮白首 & Love a lifetime \\
清平乐 & Serenade of Peaceful Joy \\
步步惊心 & Scarlet Heart \\
大明风华 & Empress of the Ming \\
金玉良缘 & Perfect Couple \\
绝代双骄 & Handsome Siblings \\
长安十二时辰 & The Longest Day in Chang'an \\
香蜜沉沉烬如霜 & Ashes of Love
\end{tabular}


《清平乐》——Serenade of Peaceful Joy

本剧以北宋为背景, 主要围绕宋仁宗的一生徐徐展开。宋朝是中国历史 上经济文化发展的高峰期, 宋仁宗在位期间, 北宋经济繁荣, 科学技术和文 化得到了很大的发展。词是宋朝最具代表性的文化结晶, 本剧以 “清平乐” 这一词牌名为剧名十分合适。再者, 本剧主要讲述只是朝堂之事和皇家的儿 女情长, “清平乐” 词内容多是伤春惜别、男女恋情, 这两者完美地契合在 一起。其英译时采用了意译的策略, 译为 Serenade of Peaceful Joy, 即 “平和 欢乐小夜曲”。该译文既传达了原剧名所要展现的恬淡平和, 同时 serenade (小 夜曲)本就来自西方, 外国受众不会因为不了解中国词文化而无法理解该剧名 的含义, 此处完美地避开了文化差异, 实在是佳译。

\section{《长安十二时辰》- - The Longest Day in Chang'an}

这部电视剧讲述了唐朝上元节前夕, 长安城陷入危局, 长安死囚张小敬 临危受命, 与李必携手在十二时辰内拯救长安的故事。原剧名虽然简洁明了, 但是存在 “长安” “时辰” 等中国特色的词汇, 其英译本摆脱了原文结构的 限制, 通过意译的手法, 将 “十二时辰” 译为 “the longest day”。十二时辰 即为一天, 日日如此, 但是英译本强调这一天是 “最长的一天”, 完美地营 造了一种紧张的气氛, 也凸显了这一天是与众不同的, 是长安城生死做关的 一天, 非常巧妙。

《香蜜沉沉烬如霜》- - Ashes of Love

该剧主要讲述了先花神之女锦受与天帝之子旭凤三世轮回的恩怨痴缠, 守望千年之恋的故事。这一剧名运用了叠词, 结构又采用七言格, 具有很强 的音律美。虽然剧名本身并没有体现剧情, 也没有明显地传达主题, 但是其 展现的意象极美。“香蜜” 让观众联想到了甜蜜的爱情, 而 “烬” 与 “霜” 指灰烬, 霜花, 则让人联想到爱恨情仇交织, 昔日甜蜜只剩下如霜花的灰烬, 冷彻心骨。Ashes of Love 直译为 “爱的灰烬”, 清晰地传达了原剧名的内涵, 而且也保留了原来的美感。不会有文化差异感。

\subsection{2. 创译}

汉弗莱(Louise Humphrey)对 “创译” 的定义是在不失去文化影响的前提 下, 摆脱对原文的依赖, 依据目的语语言、文化的要求, 对词语、创新力的 概念和观点的再创造, 实质上就是要求用不同的词语表达相同的观点 [8]。

部分古装剧剧名与主题关联性不强, 语言虽然形象, 甚至也很新鲜, 但 却无法 “照实” 传译出来, 或者直译或意译都无法形象传达原文意象, 便可 以在根据剧情以及影视剧的主题的情况下创造性地进行翻译。

下面对表 2 中具有代表性的剧名及其英译名进行分析。

\section{《陈情令》- - The Untamed}

本剧主要讲述了夷陵老祖魏无羡和含光君蓝忘机重遇, 携手探寻往年真 相, 守护百姓和平安乐的故事。剧名中 “陈情” 一词指魏无羡所持武器, 其 字面含义可解读为 “陈诉旧情” 。实际上, “陈情令” 一词到底所指为何实 在令人费解。但是, “陈情令” 三字其实承载着一定的文化信息, 容易使人 联想到晋朝文学家李密的名篇《陈情表》。故此, 虽然 “陈情令” 三字未能 
表 2. 采用创译策略的原剧名及其英译剧名

\begin{tabular}{cc}
\hline 原剧名 & 英译剧名 \\
\hline 东宫 & Good Bye, My Princess \\
陈情令 & The Untamed \\
庆余年 & Joy of Life \\
琅躯榜 & Nirvana in Fire \\
鹤涙华亭 & Royal Nirvana \\
凤涙九天 & Renascence \\
凤归四时歌 & The Legend of Jinyan \\
明月照我心 & The Love by Hypnotic \\
琉璃美人棂 & Love and Redemption \\
秦时丽人明月心 & The king's woman \\
寂寞空庭春欲晚 & Chronicle of Love \\
花落宫廷错流年 & Love in the Imperial Palace \\
那年江南那年雨 & Love Story of Court Enemies \\
少年游之一寸相思 & Love in Between \\
天雷一部之春花秋月 & The Story of Ming Lan \\
知否知否应是绿肥红瘦 &
\end{tabular}

概括剧情与主题, 但其承载的文化信息反而让中国观众产生一种熟悉感。但 是国外观众并不理解这其中蕴含的文化信息, 故无法产生类似的熟悉感。译 者摆脱了原文的束缚, 从剧情出发, 主人公魏无羡年少轻狂, 恣意潚酒, 爱 憎分明, 不为名利所缚, 不在乎世俗目光, 志在匡扶正义, 惩恶扬善, 醉酒 江湖。译者创造性将原剧名译为 “The Untamed”, 避开了文化差异这一难 题, 同时主人公 “潇酒无羁” 的形象跃然纸上。

\section{《凤唳九天》- - Renascence}

本片讲述大褚皇后姚莫心因遭陷害含恨而死, 却阴差阳错通过其妹之身 重生, 一步步开启复仇之路, 不断成长, 最终收获幸福人生。“凤凰是中国 古代传统文化中非常重要的文化形象” [9], 是祥瑞形象, 古装剧中常常用其 代表皇后, 原剧名《凤涙九天》呈现了凤凰展翅, 长啸九天, 傲视百鸟的形 象, 十分符合主角形象, 也容易引起中国观众的文化归属感。该剧名译为 Renascence, 直译为 “重生”, 充分展现了浴火重生这一主题。同时, 在西 方世界, “renascence” 即重生, 是一个非常重要的文化和宗教主题。故此, 将《凤唳九天》创造性地译为 Renascence 消除了原剧名带来的文化差异, 符 合西方观众的心理和思维方式。

《琉璃美人慜》-—Love and Redemption

在该剧中, “琉璃” 指封存魔繁星心魂的 “琉璃盏” , 也指女主人公的 琉璃心。其实, 在中国佛教文化中, “琉璃” 是佛教七宝之一, 色彩绚丽, 给人以艺术的美感和想象空间。所以, 在东方美学中, 琉璃是美的代表。而 “美人” 亦代表着美, 二者合在一起呈现的更是一种美。同时上文已经指出, 
中国人钟爱仙侠神魔之类的形象。所以说这一剧名非常符合中国观众的审美 特点和思维方式, 但是这种契合的基础就是中华文化, 这给翻译带来了巨大 挑战。译者巧妙地运用了创译策略, 从该电视剧情出发, 敏锐地捕捉到 “爱 与救赎” 这一主题。“爱” 与 “救赎” 也是西方文学作品中的永恒主题, “Love and Redemption” 这一剧名既突显了电视剧的主题, 同时也消除了原剧名中 的中西文化差异, 容易为西方观众所接受。

\subsection{3. 借译}

所谓借译，即指在翻译古装剧名的文化信息时可以套用英语语言中现成 的文化信息。语言之间既有差异，同样具有相似之处。中国古装剧剧名英译 一个重要原则就是搭建文化交流的桥梁，跨越文化差异，而借用西方文化中 对等的或是相近的形象与概念, 能够有效地实现古装剧名的翻译目的。

下面对表 3 中具有代表性的剧名及其英译名进行分析：

\section{《仙剑奇侠传 3》-CChinese Paladin 3}

这部电视剧主要讲述了景天、唐门小姐雪见、龙葵、蜀山大弟子长卿及 其爱人紫萱等一行人协助蜀山寻找五灵珠, 封印锁妖塔, 最终战胜邪剑仙, 归还三界太平。原剧名蕴含中丰富的中国文化形象，即 “仙” “侠” 形象。 在西方文化中，没有与 “仙” 相对应的概念，但是 “游侠” 形象在各国文化 中普遍存在。在西方文化中, Paladin 就完美地对应着中国的 “侠”。Paladin, 即圣骑士, 在西方文化中具有悠久的历史。自中世纪开始, 骑士道理念在欧 国各国语言文化中广泛传播, 而 Paladin一词也被用以泛指那些在史实传说中 行侠仗义的骑士。借助西方文化中的 Paladin 形象, 该英译剧名完美地解决了 中西文化差异给翻译带来的困境，给西方观众带来了一种熟悉感和认同感。

《那年花开月正圆》- - Nothing Gold Can Stay

该剧主要讲述了清末出身民间的陕西女首富周莹跌宕起伏的人生故事。 周䒯与丈夫吴聘恩爱相守, 但吴聘却不幸遭人毒手。斯人已去, 往日乐事也 如过眼云烟消散不见。此后种种, 起伏沉沦, 尝遍人生悲欢离合, 不觉感叹 那年花开时, 明月正圆, 爱人相拥。该剧名意境优美, 又散发淡淡忧伤, 朦 胧又唯美，同时采用七言格，朗朗上口。其英译版本巧妙借用美国著名诗人 Robert Frost 的同名诗句 Nothing gold can stay, 既清晰地传达了电视剧的主 题, 也保留了原文的美感, 同时还贴近译语文化。

\section{表 3. 采用借译策略的原剧名及其英译剧名}

\begin{tabular}{cc}
\hline 原剧名 & 英译剧名 \\
\hline 少女大人 & Maiden Holmes \\
萌医甜妻 & Dr. Cutie \\
热血长安 & Detective Samoyeds \\
盛唐幻夜 & An Oriental Odyssey \\
仙剑奇侠传 3 & Chines Paladin 3 \\
那年花开月正圆 & Nothing Gold Can Stay
\end{tabular}




\section{2. 表意明了类}

\subsection{1. 音译加直译}

音译法, 指 “译音代译义的方法” [10]。中国古装剧剧名常含有人名, 针 对这种情况, 可以直接翻译成汉字拼音, 保留中国特色。

直译指直接将源语言翻译成目的语, 在保持原文内容完整的情况下, 尽 量同时保持形式和结构上相近或相同。

通过表 4 可以发现, 含有人物名、朝代名的古装剧名可以采取音译加直 译的策略。人物名和朝代名运用音译，其余补充成分采用直译。如《䒠月传》

《解忧公主》《雍正王朝》运用音译加直译策略分别译为 Legend of Mi Yue, Princess Jieyou 和 You Zheng Dynasty, 英译本既保留了原剧名的特色, 又通 俗易懂，达到了跨文化传播的效果。

\subsection{2. 减译}

减译法, 也称减词法, 其翻译效果为 “减词不减意” [11]。上文提出, 中 国古装剧名也具备表意明了的特点, 但是实际上, 许多表意通达的古装剧名 中还是含有许多累赘的修饰成分, 这主要是因为汉语追求音律美, 模糊美。 根据英美古装剧名简洁明了的特点, 在翻译中国古装剧名时, 可以删减不重 要的没有实质意义的修饰成分, 只保留最重要的成分, 简洁直白地传递原文 信息，同时消除文化差异带来的麻烦。

如表 5 所示, 《神探包青天》这一剧名中最重要就是 “侦探” 和 “包拯” 这两个信息, 汉语语言主体意识强, 喜欢使用一些修饰词, 用以加强表意效 果, 如描述查案之人技艺高超则称其为 “神探” , 而英语语言客体意识强, 更追求客观描述, 所以在翻译此剧名时删去 “神” 字是合适的, 其英译本 Detective Justice Bao 简洁明了, 客观实在。同样的, 《人间烟火花小嶎》这 一剧名主要就是在表达 “花小厨” ，即一位姓花的小厨娘。原剧名加上 “人 间烟火”一词, 构成七言结构, 具有音律美, 同时 “人间烟火”与 “厨师” 在意义上有联系之处, 中国观众对于 “不食人间烟火” 这一表达也耳熟能详,

表 4. 采用音译加直译策略的原剧名及其英译剧名

\begin{tabular}{cc}
\hline 原剧名 & 英译剧名 \\
\hline 晧镧传 & The Legend of Hao Lan \\
苹月传 & Legend of Mi Yue \\
芸汐传 & Legend of Yun Xi \\
班淑传奇 & Ban Shu Legend \\
陆贞传奇 & Legend of Lu Zhen \\
解忧公主 & Princess Jieyou \\
武神赵子龙 & God of War, Zhao Yun \\
铁齿铜牙纪晓岗 & The Eloquent Ji Xiaolan \\
雍正王朝 & Yong Zheng Dynasty \\
楚汉传奇 & Legend of Chu and Han \\
\hline
\end{tabular}


表 5. 采用减译策略的原剧名及其英译剧名

\begin{tabular}{cc}
\hline 原剧名 & 英译剧名 \\
\hline 神探包青天 & Detective Justice Bao \\
拜见宫主大人 & Your Highness \\
人间烟火花小厨 & Chef Hua \\
大汉贤后卫子夫 & The Virtuous Queen of Han \\
山海经之上古密约 & Guardians of the Ancient Oath \\
大军师司马懿之虎啸龙吟 & Growling Tiger, Roaring Dragon \\
\hline
\end{tabular}

所以 《人间烟火花小㕑》这一剧名虽然含有没有实质意义的词, 却比 “花小 㕑” 这一剧名更容易为中国观众所接受。其英译本删减 “人间烟火”一词, 译为 “Chef Hua”, 简洁明了, 重点突出, 容易让外国观众理解。

\section{4. 结语}

在中国文化走出去的战略背景下, 以影视为载体进行文化传播是一种行 之有效的策略。而古装剧汇集了丰富的中国古典文化, 研究古装剧翻译具有 重要意义。古装剧名作为一种特殊的文本，不只承担着信息传递的作用，更 承担着审美和广告宣传的功能。根据中国与英美古装剧名的特点, 本文提出 中国古装剧名翻译的总原则为由繁入简, 由含蓄到简明。如果剧名委婉含蓄, 可进行意译, 借译, 创译。如果剧名表意明了, 可采用音译加直译一一减译 的策略。虽然本文针对不同特点的古装剧名提出了相应的翻译策略, 但是对 于文化差异的研究还不够深入, 剧名的分类较为宽泛, 未来还可以对此进行 进一步研究, 也希望本文能够为中国古装剧译制研究带来一些新启发, 对影 视文化交流有所禆益。

\section{Conflicts of Interest}

The authors declare no conflicts of interest regarding the publication of this paper.

\section{References}

[1] 贺莺. 电影片名的翻译理论和方法[J]. 外语教学, 2001, 22(1): 56-60.

[2] 连淑能. 论中西思维方式[J]. 外语与外语教学, 2002(2): 40-46.

[3] 冯梅. 重命名翻译是另类翻译法么? 一一兼与康志洪、何自然等商榷 [J]. 重庆理工 大学学报(社会科学), 2013, 27(12): 99-104.

[4] 朱怡珓. 东方审美与西方想象: 玄幺题材电视剧中角色形象的塑造[J]. 中国电视, 2017(2): 52-56.

[5] 王燕灵. 论国产电视剧“走出去”战略[J]. 新闻传播, 2015(23): 25-27.

[6] 刘志, 马芳菲. 试论优秀国产剧的海外市场拓展[J]. 韶关学院学报, 2016, 37(5): 69-73.

[7] 罗竹风. 汉语大词典: 第七卷上 $[M]$. 上海: 上海辞书出版社, 1993: 646 .

[8] [美]劳伦斯.韦努蒂(Lawrence Venuti). 译者的隐身 $[M]$. 上海: 上海外语教育出版 
社, 2004.

[9] 尤伊凡. 先秦两汉凤凰形象研究[J]. 文化学刊, 2020(2): 240-242.

[10] 王丽. 音译法使用中的“对等”原则 [J]. 山东外语教学, 2010, 31(4): 110-112.

[11] 许渊冲. 文学翻译: $1+1=3$ [J]. 外国语(上海外国语学院学报), 1990(1): 8-12.

\section{Appendix (Abstract and Keywords in Chinese)}

\section{文化差异视角下中国古装剧剧名英译}

摘要：古装剧中蕴含着丰富的中国文化因素, 但古装剧名英译中存在着 不可逃避的问题，即文化差异。中国古装剧剧名总体而言具有两大特点，委 婉含蓄和表意明了, 而英美古装剧剧名则是通畅简明。故此, 本文提出, 中 国古装剧名英译的主导原则为, 由繁入简, 由模糊虚幻到具体实在。本文将 中国古装剧剧名分为两类, 针对性地提出具体的翻译策略。委婉含蓄的剧名 选择意译、创译和借译策略; 表意明了的剧名宜采用音译加直译和减译策略。

关键词: 古装剧名, 中国文化, 文化差异 